\title{
Mandibular Advancement Devices for OSA: An Alternative to CPAP?
}

\author{
Claire E. Francis · Tim Quinnell
}

Received: July 21, 2020 / Accepted: October 19, 2020 / Published online: November 10, 2020

(C) The Author(s) 2020

\begin{abstract}
Obstructive sleep apnea (OSA) is a prevalent condition causing unrefreshing sleep and excessive daytime sleepiness. It has individual socioeconomic impacts and, through association with increased risk of road traffic accidents, diabetes, and cardiovascular disease, OSA is a public health issue. Continuous positive airway pressure (CPAP) is the first-line treatment for moderate-to-severe OSA. It is effective in improving excessive daytime sleepiness and quality of life. There is also evidence that CPAP therapy has cardiovascular benefits although nature and extent remain uncertain. Despite its benefits, a significant proportion of patients are unable to tolerate CPAP. There are also patients with mild but symptomatic disease, for whom CPAP is usually not available or appropriate, so there is a need for other treatment options. Mandibular advancement devices (MADs) offer an effective alternative to CPAP and can improve daytime symptoms and quality of life.
\end{abstract}

There are many devices available, representing a range of complexity and cost. It is challenging to properly evaluate the effectiveness of this ever-evolving range. The more basic MADs are cheaper and more accessible but are less well tolerated. More complex devices are better tolerated and may be more effective. However, they are more expensive and often require dental expertise, so access is more limited. Efforts continue to try to improve accessibility to effective MAD therapy. Alongside increasing awareness, this may be facilitated by developing and refining devices that could be fitted by nondental clinicians, and potentially by patients themselves. Research efforts need to focus on determining how to efficiently identify patients who are likely to respond to MAD therapy, so as to improve clinical and cost-effectiveness of OSA therapy overall.

Keywords: CPAP alternatives; Mandibular advancement device; Obstructive sleep apnea

C. E. Francis · T. Quinnell ( $\square)$

Royal Papworth Hospital NHS Foundation Trust, Cambridge, UK

e-mail: tim.quinnell@nhs.net 


\section{Key Summary Points}

Mandibular advancement devices offer an alternative to CPAP therapy in treating obstructive sleep apnea.

There is a wide range of devices available, representing a range of complexity, cost, and accessibility.

Challenges remain regarding predicting response to treatment and optimizing protrusion.

Future research should focus on response prediction and exploring the potential of combining MAD with other treatments.

\section{DIGITAL FEATURES}

This article is published with digital features, including a summary slide, to facilitate understanding of the article. To view digital features for this article go to https://doi.org/10.6084/ m9.figshare.13103420.

\section{BACKGROUND}

Obstructive sleep apnea (OSA) involves repeated excessive narrowing of the upper airway during sleep. This causes pauses in breathing and oxygen desaturations associated with blood pressure and heart rate fluctuations [1], leading to unrefreshing sleep and excessive daytime sleepiness. A recent literature review of global prevalence estimated that nearly 1 billion adults aged 30-69 years could have OSA, with 425 million suffering from moderate-to-severe OSA [2]. Factors associated with increased risk of OSA include male gender, increasing age, and increased BMI [3]. Untreated OSA has been associated with diabetes [1], cardiovascular disease [4], and road traffic accidents [5], making effective treatment a public health issue.

There are several different treatment options available for OSA and selecting the best treatment for an individual is not always straightforward. OSA is a heterogeneous condition and symptom burden correlates poorly with objective measures of severity obtained from sleep studies [6]. At the extremes, patients with severe OSA can be asymptomatic, whereas others with only mild disease can suffer debilitating daytime sleepiness. It can therefore be difficult to predict which patients will benefit from treatment. This brief narrative review will discuss the current evidence and guidelines on treating OSA with a mandibular advancement device (MAD) as an alternative to the current gold-standard treatment. This article is based on previously conducted studies and does not contain any studies with human participants or animals performed by any of the authors.

\section{CPAP AS THE FIRST-LINE TREATMENT}

Continuous positive airway pressure (CPAP) is the current first-line treatment for moderate-tosevere OSA [7]. It greatly improves objective indices of OSA (e.g., apnea-hypopnea index$\mathrm{AHI} /$ hour), and has significant beneficial effects on symptoms including daytime sleepiness and quality of life [8]. There is also some evidence that CPAP has beneficial cardiovascular effects, with studies showing improvements in blood pressure [9-11]. However, the literature is inconsistent regarding the extent of potential cardiovascular benefit conferred by CPAP. For example, the multicenter prospective controlled SAVE study failed to show a significant positive effect of CPAP on cardiovascular outcomes in patients with moderate-to-severe OSA and a history of coronary or cerebrovascular disease [12].

A problem for the SAVE study in common with many other CPAP clinical trials was low CPAP adherence, which averaged $3.3 \mathrm{~h}$ per night. This is below the widely accepted threshold for adequate adherence, which is $4 \mathrm{~h}$ a night [13]. Achieving satisfactory CPAP adherence is challenging, and published rates are highly variable, with $46-83 \%$ of users found to be non-adherent to treatment [13]. The 4-h threshold is semi-arbitrary but there is evidence 
for higher adherence translating into greater benefits, in terms of subjective (Epworth Sleepiness Scale-ESS) and objective (multiple sleep latency test-MSLT) daytime sleepiness, functional status, and blood pressure [14]. No single factor has been consistently identified as predictive of poor adherence. However, issues frequently highlighted by patients include high CPAP pressure, poor mask fit, disturbance of bed partner, and claustrophobia [13]. Another barrier to many OSA sufferers potentially benefiting from CPAP is that it is currently only recommended as the first-line treatment for patients with moderate-to-severe OSA (AHI > $15 / \mathrm{h}$ ) [7]. Data recently produced by the MERGE study showed that CPAP also improves quality of life in patients with mild OSA [15], and it is possible that future clinical guidelines will be influenced by these findings.

Given the limitations of CPAP therapy in terms of tolerance and availability, there is a need for alternative treatment options for patients with milder OSA and for those with more severe disease who cannot tolerate CPAP. The key challenges for OSA management include deciding which patients to treat, which treatment to choose for the individual, and then supporting patients to adhere to therapy once it has been started.

\section{ALTERNATIVES TO CPAP}

Lifestyle measures should be considered in all patients diagnosed with OSA. This will be casespecific but may include advice on weight loss, sleep hygiene, sleep position, and exercise levels, and moderation of exacerbating factors such as alcohol intake and smoking [16]. For predominantly positional OSA, devices which aim to stop patients sleeping on their back have been piloted with mixed results [17]. Significant weight loss can eliminate the need for treatment, or reduce the pressure required to effectively control OSA, which could help to improve CPAP tolerance [16]. There is also evidence that, when combined with CPAP, weight loss can improve insulin sensitivity, dyslipidemia, and blood pressure significantly more than can be achieved by either intervention alone [18]. In relevant cases, bariatric surgery can also be used to improve OSA outcomes [19]. Exercise training may also be a useful adjunct to OSA treatment in reducing $\mathrm{AHI}$ and improving daytime sleepiness [20].

In addition to lifestyle measures, there are several alternatives to CPAP therapy for OSA, although few are used widely in clinical practice. Mandibular advancement device therapy is the most extensively studied CPAP alternative [21]. Clinical guidelines continue to be updated, but most currently recommend MAD as the first-line treatment option in mild OSA $[7,21,22]$. There is some variability in the area of moderate OSA, while MAD therapy is more consistently recommended as the second-line option to CPAP in severe OSA. There is increasing interest in other non-CPAP therapies, including positional devices [17], hypoglossal nerve stimulation [23], and transcutaneous electrical nerve stimulation [24]. As yet, the evidence is not strong enough to consider these except in select groups of patients for whom CPAP and/or MAD therapy have failed or are not appropriate.

\section{TYPES OF MADS}

Mandibular advancement devices protrude the mandible forward and in so doing also advance the tongue. This moves the upper airway further away from critical narrowing, probably by increasing pharyngeal dimensions and reducing collapsibility [25], although the exact mechanisms remain unclear and may vary between individuals. Using a MAD has been shown to increase total pharyngeal area, particularly at the level of the soft palate, which has been associated with a decrease in the pressure drop throughout the upper airway during inspiration, helping to prevent apneas [26]. There is some evidence to suggest that the improvement in pharyngeal collapsibility with MAD therapy occurs in a dose-dependent manner (i.e., with greater protrusion) [27].

There are many different MADs available, representing a range of complexity, cost, and therefore accessibility. Common to all MAD is that they must fit to the upper and lower teeth 
in order to advance and anchor the mandible on the maxilla. Devices can be one-piece ("monobloc"), where the upper and lower components are fused or two-piece devices which usually come apart but in order to work are attached to each other when worn. Attachment designs vary considerably but they are invariably adjustable to allow titration of mandibular protrusion according to tolerance and efficacy. The most basic type of MAD is the thermoplastic monobloc, which is usually sourced directly without input from a dental specialist. Patients immerse the device in boiling water to soften it and then mold it to their teeth, determining the degree of mandibular advancement in the process. Customized devices are generally more sophisticated, although still wide-ranging. Almost all are provided by dental or maxillofacial specialists, who obtain a patient's dental impression to create and fit the bespoke MAD, now often manufactured by a commercial provider. While some customized devices are monoblocs, two-piece adjustable MADs are increasingly common and usually preferred by specialist dental providers.

The technology associated with MAD design is continually evolving. Ongoing innovation is bridging the gap between patient-molded and specialist-fitted MADs. Thermoplastic and adjustable technologies are being combined in attempts to reconcile the requirements for access and affordability with comfort, durability, and efficacy. While this may be good for patients, the ever-increasing heterogeneity of devices makes it challenging for research to keep up to date and for front-line users to make sense of the options. This, combined with variable access to funding for MAD therapy, makes what is essentially a simple concept a complex area of OSA treatment.

\section{EFFECTIVENESS OF MAD THERAPY COMPARED TO CPAP}

The heterogeneity of MAD therapy is an important factor in the variability in efficacy, patient tolerance, and cost-effectiveness [25]. Compared to no treatment, MADs of varying complexity can significantly reduce AHI and improve daytime sleepiness in patients with OSA $[28,29]$. There is evidence that MAD therapy can improve blood pressure [30], particularly in patients with baseline hypertension [31-33]. However, as with CPAP, there is no strong evidence that MAD therapy has beneficial effects on other co-morbidities associated with OSA. MADs are generally not as effective as CPAP at reducing AHI. In a meta-analysis, Sharples et al. reported that data from 13 trials (746 patients) showed CPAP reduced AHI on average by $7.03 / \mathrm{h}(p<0.001)$ more than MAD therapy [34]. This effect may be related to baseline AHI, as most studies comparing CPAP with MAD therapy have been conducted in patients with moderate-to-severe OSA [34], so MADs may nonetheless be the appropriate firstline treatment option in mild OSA.

The greater efficacy of CPAP may be offset by better tolerance of, and therefore adherence to, MAD therapy [32]. This introduces the concept of mean disease alleviation, whereby there is net equivalent AHI reduction due to a less efficacious MAD being worn for longer overnight than CPAP [32]. This is thought to be one reason why MADs appear to be as effective as CPAP at improving excessive daytime sleepiness [28]. A randomized crossover study which compared 1 month of titrated MAD therapy with optimized CPAP found that both treatments improved daytime sleepiness, driving simulator performance and quality of life to a similar extent [32]. Self-reported compliance with CPAP was $5.2 \mathrm{~h} /$ night compared to $6.5 \mathrm{~h} /$ night for MAD therapy $(p<0.0001)$. A meta-analysis found that both MADs and CPAP significantly reduced systolic and diastolic blood pressure with no difference in treatment effects, which was proposed to be due to greater MAD adherence [33]. Younger age ( $<60$ years), uncontrolled blood pressure, and severe OSA-related desaturations have been identified as positive predictive factors for exhibiting a favorable blood pressure response to treatment with either CPAP or MAD [11]. Long-term data suggest that after 10 years of usage, both CPAP and MAD therapy significantly reduced AHI compared to baseline, suggesting that both provide stable long-term treatment options [35]. 


\section{EFFECTIVENESS OF DIFFERENT TYPES OF MADS}

There are a limited number of randomized controlled trials comparing different MADs. One randomized crossover study of 35 patients with mild-to-moderate OSA compared a basic thermoplastic monobloc with a custom-made, adjustable, specialist-fitted MAD. While both improved symptoms compared to baseline, the custom-made MAD was superior in terms of patient tolerance, AHI improvement, and reduction of daytime sleepiness [36]. Patients used each device for 3 months with a 2-week washout period. AHI was reduced by at least $50 \%$ in $96 \%$ of patients when wearing the custom-made MAD, whereas only $64 \%$ showed the same AHI reduction while wearing the thermoplastic MAD $(p<0.001)$. Sixty-four percent of patients experienced a normalization of AHI $(<5 / \mathrm{h})$ using the custom-made MAD, while only $24 \%$ saw a normalization when wearing the ready-made MAD $(p<0.05)$.

The TOMADO study compared three different types of monobloc MAD [37]. Ninety patients were randomized in a crossover design to wear a thermoplastic device, a 'semi-bespoke' device, and also a custom-made device that was made and fitted by a dentally qualified maxillofacial team. The semi-bespoke device used thermoplastic technology to enable patients to take their own dental impression, which was used by a manufacturer to produce a custommade end-product independent of specialist dental input. All three types of MAD significantly improved AHI and ESS compared to baseline, and all devices were cost-effective. However, the thermoplastic device was less comfortable and fell out more often, undermining adherence and making it the least popular device. The semi-bespoke and specialist provided MADs were similar in terms of clinical effectiveness, meaning that the cheaper and more accessible semi-bespoke device was found to be the most cost-effective option.

Absent from TOMADO was an adjustable MAD. The ability to titrate mandibular protrusion according to symptom control and comfort has, to some extent, distinguished custom-made devices from thermoplastic alternatives. This has supported expert recommendations advocating custommade devices despite the scarcity of high-level comparative evidence. Device innovation has led to thermoplastic technology being combined with adjustability [38]. Recently, Pepin et al. compared a custom-made adjustable MAD to a thermoplastic adjustable device [39]. In this randomized controlled trial, 198 patients with moderate-to-severe OSA who refused or did not tolerate CPAP wore either a thermoplastic heatmolded adjustable MAD or a two-piece custommade acrylic MAD for 2 months. The thermoplastic device was found to be non-inferior to the custom-made device in terms of patient tolerance and effectively treating OSA, including quality of life and symptom benefits. A dentist fitted and supervised the titration of both MADs throughout the study, so there remain questions regarding accessibility and cost for the more basic device.

To what extent specialist dental input influences outcomes is unclear. One factor that a dentist may influence is the degree of mandibular advancement achieved. While more may be considered better in terms of efficacy, this can undermine device tolerance. There is significant variability in the literature regarding degree of protrusion used, with studies aiming for between 50 and $100 \%$ of maximal mandibular protrusion [27, 31, 40]. Several different titration protocols have been trialed but there is no gold-standard method or agreed optimum degree of protrusion [41] and patients sometimes struggle to tolerate protocolized titration [42]. Using polysomnography (PSG) to remotely titrate the degree of protrusion has been shown to be an effective method of optimizing apnea control [43-45]. A recently published stepwise titration protocol for treating positional OSA suggested that $75 \%$ maximal protrusion provided the optimal balance between benefit and tolerance for most patients [46]. In truth, the optimal protrusive position will be individual, which is why patients can also effectively titrate their MAD according to symptoms and comfort alone [32, 41]. 


\section{PREDICTING RESPONSE TO MAD THERAPY}

A key challenge in using MADs to treat OSA is predicting response to treatment. Baseline severity of OSA is a poor predictor of response to MAD therapy, as some patients with mild or moderate OSA can show no improvement while other patients with severe disease respond well [47]. Factors such as obesity [48], older age [49], male gender [50], and non-positional OSA [51] are imprecise predictors of poor response to MAD therapy. One study found positional OSA to be the only independent predictor of successful reduction in AHI (defined in this study as $>50 \%$ reduction in $\mathrm{AHI}$ and residual $\mathrm{AHI}<$ $10 / \mathrm{h}$ ) after 5 weeks of using a custom-made monobloc MAD [52].

Tongue position has been suggested to predict response. Patients with a posteriorly located tongue reported to be significantly more likely to exhibit higher treatment efficacy [53]. These traits can be measured using drug-induced sleep endoscopy (DISE) [54]. However, this is an expensive, invasive, and time-consuming procedure, which is not practical for (or generalizable to) routine clinical practice.

Some of the variability in response to MAD therapy may relate to the extent to which pharyngeal (collapsibility and muscle compensation) and non-pharyngeal (loop gain, arousal threshold, and ventilatory response to arousal) traits contribute toward OSA pathogenesis [55]. Non-pharyngeal traits in particular are complex, and details are beyond the scope of this article. Put simply, arousal threshold refers to the threshold at which airway obstruction causes cortical arousal. Loop gain refers to the propensity of a system that is controlled by feedback loops to develop unstable behavior. The higher the loop gain for an individual's respiratory system, the more unstable the control of breathing may be in response to any breathing disturbance [56].

Preliminary work has explored the potential for PSG results to evaluate non-anatomical pharyngeal traits. Detailed explanation of the development and validation of the techniques used to derive pharyngeal and non-pharyngeal characteristics from PSG data are described elsewhere $[57,58]$. Edwards et al. (2016) derived these parameters using CPAP pressure changes in 14 patients $(\mathrm{AHI}>10)$ sleeping with and without a MAD [59]. They found lower airway collapsibility and lower loop gain to be independent predictors of MAD efficacy (defined by $\% A H I$ reduction). The efficacy of MAD therapy appeared to be mediated via reduction of upperairway collapsibility and narrowing or reversal of the gap between the ventilation threshold for arousal and that at which pharyngeal dilator activity increases to maintain airway patency.

Recently, Bamagoos et al. tested a more pragmatic approach using PSGs undertaken on 93 patients $(\mathrm{AHI}>=20)$ on and off MAD therapy drawn from three clinical research studies [55]. The same characteristics as those examined by Edwards et al. were derived without using CPAP. These less-precise derivations were applied to response prediction. Multivariate regression analyses identified pharyngeal (moderate airway collapsibility and lower pharyngeal dilator muscle response to airflow limitation) and non-pharyngeal (lower loop gain, higher arousal threshold) features that were associated with greater MAD efficacy. The investigators present this technique as a potential means of identifying those patients with moderate or severe OSA who would be more likely to respond to MAD therapy. The data thus far suggest it has poor negative predictor power (so it can't reliably identify nonresponders), but it might help determine the order of play (CPAP or MAD first). More extensive and prospective controlled trials are needed to properly establish the accuracy of this approach. If reliability were established, then it might, with refinements, be generalizable and useful to clinical sleep services, but only those that use PSG as their entry-level diagnostic test.

\section{DISADVANTAGES OF MAD THERAPY}

The variable and difficult-to-predict effectiveness of MAD therapy is a key obstacle to their overall clinical usage. Although benefits to sleepiness and other health outcomes appear 
similar to CPAP [32, 34], concerns about potential harm from undertreating OSA may hamper their wider uptake. As such, they are usually only recommended as second-line treatment for patients with moderate-to-severe OSA who have failed CPAP and, if needed, alongside lifestyle measures in mild OSA $[7,21]$. If progress can be made in improving our ability to reliably and cheaply predict MAD responders, and device innovation continues to improve the quality of and accessibility to treatment, then MAD therapy may become more widespread.

Contraindications to MAD therapy are another barrier to treatment. In one study of 100 patients with OSA, 34 were found to have contraindications to using a MAD [60]. Guidelines on contraindications are based largely on expert consensus and can be vague on details $[16,61]$. Most clinicians would agree that patients with severe daytime sleepiness and/or severe oxygen desaturations should be advised to try CPAP first, and only resort to trying a MAD if they could not tolerate CPAP or other positive pressure options. Broadly, MADs are only an option for those patients whose dentition, gums, and temporomandibular function are healthy and sufficiently robust. There need to be sufficient healthy teeth (or implants) to allow adequate device retention, but precisely how many teeth are required in each arch is somewhat arbitrary, and may be influenced by device sophistication. In the TOMADO study, physicians screening patients for eligibility for the trial followed expert advice to exclude those who were missing more than two teeth in each arch, but this was relatively conservative. Untreated periodontal disease is considered a contraindication, as there is a risk of teeth being moved by MAD therapy [62]. Some previously considered absolute contraindications may be relative and mitigated by other interventions. Temporomandibular disorder (TMD) has been highlighted as the most prevalent condition that may be aggravated by MAD therapy $[21,63]$. Mandibular exercises have been shown to help alleviate the negative impacts of MAD therapy on this disorder [63]. A recent metaanalysis by dental experts suggested that TMD should not necessarily be a contraindication, as patients with pre-existing signs and symptoms of TMD did not experience significant worsening of TMD while using a MAD [64].

Concerns about contraindications to and side effects from MAD therapy feed into the debate about the role of dental specialists. Offthe-shelf MADs do not usually require specialist dental input, although patients may seek help and some devices come with advice on dental oversight. If patients have a bad experience with a basic device, this can put them off persevering with MAD therapy. However, if MAD therapy in the UK were confined to custom-made devices provided by those maxillofacial and dental specialists who are interested in providing it, then access would be more limited than it already is. In an online survey of 62 sleep centers from across the UK, $52 \%$ were unable to access NHS-funded MAD therapy for their patients. Of those that could, 50\% had access to custom-made MAD therapy provided by a dentist or maxillofacial colleague (unpublished survey data). Dental sleep expertise is still developing in the UK. This may be hampered by uncertainty regarding NHS funding, although the situation could change depending on the recommendations of new UK national guidelines (National Institute for Health and Care Excellence) currently being drafted. One argument for involving a dental specialist is to check a patient's suitability for MAD therapy, given the potential risks of implementing it in someone with poor dentition. Experience from TOMADO suggests that these risks could be mitigated with simple screening procedures. In that study, patients were initially screened for eligibility by one of two respiratory physicians who applied basic dental exclusion criteria provided by a maxillofacial specialist. Only two of 92 patients considered suitable for MAD therapy by a respiratory physician were subsequently deemed ineligible by the maxillofacial specialist, due to poor oral hygiene and tooth decay [37].

There is limited evidence on the long-term effects of regular MAD use on dental positioning. One study reported significant (but clinically irrelevant) changes in dentition (displacement of the incisors), in 15 patients with moderate OSA after 6 months of using a 
twin block MAD [65]. A recent meta-analysis found significant change in overbite and overjet with MAD use, but the authors acknowledge that the included studies had significant risk of bias [66]. Existing evidence suggests that MAD usage is associated with long-term side effects. However, their extent and importance remain unclear and need to be considered against the benefits of treating OSA. Generally, patients already using a MAD should be advised to inform their dentist and to have regular dental check-ups. If there is any concern regarding whether dentition is suitable, they should be instructed to see a dentist before using a MAD.

Another limiting factor in MAD therapy is patient preference. As with CPAP, some patients cannot tolerate wearing a MAD. Even in the absence of serious dental side effects, commonly reported problems include the device falling out overnight, oral dryness, excessive saliva production, and masticatory muscle discomfort [67]. The TOMADO study found that adherence was poor for the basic thermoplastic device, due to discomfort and poor device retention. However, there were no significant differences in side-effect profiles or adherence between the semi-bespoke and the custommade monobloc device [37]. A possible advantage of having a range of devices on the market means a wide choice may be available to patients at initial diagnosis. In reality, this will be limited by what is advised or available through the publicly funded healthcare system and what the patient is willing or able to pay privately. The process of accessing MAD therapy can also take time and initial negative experiences may cause some patients to disengage early from this pathway. As CPAP becomes simpler and masks become more comfortable, it may be that for some patients who were previously unable to tolerate it, CPAP becomes a better option, negating the need to consider an alternative.

\section{FUTURE DIRECTIONS}

If further progress can be made in refining the ability to predict response to MAD therapy, then this could improve both its clinical and cost-effectiveness. Potential responders could be offered MAD therapy while predicted 'non-responders' could be offered CPAP, try other alternatives, or simply be advised to pursue conservative treatment as appropriate. There needs to be a continuing focus on comparing different types of MAD, and on developing and testing devices which bridge the gap between basic 'off-the-shelf' options and custom-made MADs. Another potential role for MADs that is in need of further exploration is their use in combination therapy. There is limited evidence that combining a MAD simultaneously with CPAP may help improve adherence to treatment, possibly by reducing the CPAP pressure required to effectively treat OSA $[68,69]$. Another combination in need of further exploration in selected patients is the use of a MAD with positional therapy, for patients with breakthrough OSA when using one or other of these treatments [70].

\section{CONCLUSIONS}

While CPAP is still the first-line treatment for moderate-to-severe OSA, MADs are an effective option for patients who are intolerant of CPAP and those with milder disease. Advances in design are providing a wider range of choices for patients, narrowing the divide between the lesseffective over-the-counter devices and high-end bespoke adjustable MADs. Given the progress being made with innovative off-the-shelf devices, it is possible that dentally fitted custommade MADs will not be required for many patients with OSA who choose to try this treatment in the future. Ongoing comparative research will need to accompany innovations in MAD technology. Work is also needed to continue to develop reliable and accessible techniques for predicting response to MAD therapy. In these ways, we could improve our ability to provide precision medicine to patients with OSA. 


\section{ACKNOWLEDGEMENTS}

Funding. No funding or sponsorship was received for this study or publication of this article.

Authorship. All named authors meet the International Committee of Medical Journal Editors (ICMJE) criteria for authorship for this article, take responsibility for the integrity of the work as a whole, and have given their approval for this version to be published.

Disclosures. Claire E. Francis and Tim Quinnell have no financial disclosures or conflicts of interests to declare.

Compliance with Ethics Guidelines. This article is based on previously conducted studies and does not contain any studies with human participants or animals performed by any of the authors.

Open Access. This article is licensed under a Creative Commons Attribution-NonCommercial 4.0 International License, which permits any non-commercial use, sharing, adaptation, distribution and reproduction in any medium or format, as long as you give appropriate credit to the original author(s) and the source, provide a link to the Creative Commons licence, and indicate if changes were made. The images or other third party material in this article are included in the article's Creative Commons licence, unless indicated otherwise in a credit line to the material. If material is not included in the article's Creative Commons licence and your intended use is not permitted by statutory regulation or exceeds the permitted use, you will need to obtain permission directly from the copyright holder. To view a copy of this licence, visithttp://creativecommons.org/licenses/by$\mathrm{nc} / 4.0 /$.

\section{REFERENCES}

1. Jordan AS, McSharry DG, Malhotra A. Adult obstructive sleep apnoea. Lancet. 2014;383(9918): 736-47. https://doi.org/10.1016/S01406736(13)60734-5.

2. Benjafield AV, Ayas NT, Eastwood PR, et al. Estimation of the global prevalence and burden of obstructive sleep apnoea: a literature-based analysis. Lancet Respir Med. 2019;7(8):687-98. https:// doi.org/10.1016/S2213-2600(19)30198-5.

3. Senaratna CV, Perret JL, Lodge CJ, et al. Prevalence of obstructive sleep apnea in the general population: a systematic review. Sleep Med Rev. 2017;08(34):70-81. https://doi.org/10.1016/j.smrv. 2016.07.002.

4. Dong JY, Zhang YH, Qin LQ. Obstructive sleep apnea and cardiovascular risk: meta-analysis of prospective cohort studies. Atherosclerosis. 2013;229(2):489-95. https://doi.org/10.1016/j. atherosclerosis.2013.04.026.

5. Ellen RL, Marshall SC, Palayew M, Molnar FJ, Wilson KG, Man-Son-Hing M. Systematic review of motor vehicle crash risk in persons with sleep apnea. J Clin Sleep Med. 2006;2(2):193-200.

6. Edwards BA, Redline S, Sands SA, Owens RL. More than the sum of the respiratory events: personalized medicine approaches for obstructive sleep apnea. Am J Respir Crit Care Med. 2019;200(6):691-703. https://doi.org/10.1164/rccm.201901-0014TR.

7. National Institute of Health and Care Excellence (2008) Continuous positive airway pressure for the treatment of obstructive apnoea/hypopnea syndrome. Technology appraisal guidance www.nice. org.uk/guidance/ta139.

8. Patil SP, Ayappa IA, Caples SM, Kimoff RJ, Patel SR, Harrod CG. Treatment of adult obstructive sleep apnea with positive airway pressure: an American Academy of Sleep medicine clinical practice guideline. J Clin Sleep Med. 2019;15(2):335-43. https:// doi.org/10.5664/jcsm.7640.

9. Martínez-García MA, Capote F, Campos-Rodríguez $\mathrm{F}$, et al. Effect of CPAP on blood pressure in patients with obstructive sleep apnea and resistant hypertension: the HIPARCO randomized clinical trial. JAMA. 2013;310(22):2407-15. https://doi.org/10. 1001/jama.2013.281250.

10. Pepperell JC, Davies RJ, Stradling JR. Systemic hypertension and obstructive sleep apnoea. Sleep Med Rev. 2002;6(3):157-73. https://doi.org/10. 1053/smrv.2001.0189. 
11. Pengo MF, Soranna D, Giontella A, et al. Obstructive sleep apnoea treatment and blood pressure: which phenotypes predict a response? A systematic review and meta-analysis. Eur Respir J. 2020. https://doi.org/10.1183/13993003.01945-2019.

12. McEvoy RD, Antic NA, Heeley E, et al. CPAP for prevention of cardiovascular events in obstructive sleep apnea. N Engl J Med. 2016;375(10):919-31. https://doi.org/10.1056/NEJMoa1606599.

13. Weaver TE, Grunstein RR. Adherence to continuous positive airway pressure therapy: the challenge to effective treatment. Proc Am Thorac Soc. 2008;5(2): 173-8. https://doi.org/10.1513/pats.200708-119MG.

14. Weaver TE, Maislin G, Dinges DF, et al. Relationship between hours of CPAP use and achieving normal levels of sleepiness and daily functioning. Sleep. 2007;30(6):711-9. https://doi.org/10.1093/ sleep/30.6.711.

15. Wimms AJ, Kelly JL, Turnbull CD, et al. Continuous positive airway pressure versus standard care for the treatment of people with mild obstructive sleep apnoea (MERGE): a multicentre, randomised controlled trial. Lancet Respir Med. 2020;8(4):349-58. https://doi.org/10.1016/S2213-2600(19)30402-3.

16. Epstein LJ, Kristo D, Strollo PJ, et al. Clinical guideline for the evaluation, management and long-term care of obstructive sleep apnea in adults. J Clin Sleep Med. 2009;5(3):263-76.

17. Srijithesh PR, Aghoram R, Goel A, Dhanya J. Positional therapy for obstructive sleep apnoea. Cochrane Database Syst Rev. 2019;5:CD010990. https://doi.org/10.1002/14651858.CD010990. pub2.

18. Chirinos JA, Gurubhagavatula I, Teff K, et al. CPAP, weight loss, or both for obstructive sleep apnea. N Engl J Med. 2014;370(24):2265-75. https://doi. org/10.1056/NEJMoa1306187.

19. de Raaff CAL, de Vries N, van Wagensveld BA. Obstructive sleep apnea and bariatric surgical guidelines: summary and update. Curr Opin Anaesthesiol. 2018;31(1):104-9. https://doi.org/10. 1097/ACO.0000000000000542.

20. Iftikhar IH, Bittencourt L, Youngstedt SD, et al. Comparative efficacy of CPAP, MADs, exercisetraining, and dietary weight loss for sleep apnea: a network meta-analysis. Sleep Med. 2017;02(30): 7-14. https://doi.org/10.1016/j.sleep.2016.06.001.

21. Ramar K, Dort LC, Katz SG, et al. Clinical practice guideline for the treatment of obstructive sleep apnea and snoring with oral appliance therapy: an update for 2015. J Clin Sleep Med. 2015;11(7): 773-827. https://doi.org/10.5664/jcsm.4858.
22. Randerath WJ, Verbraecken J, Andreas S, et al. NonCPAP therapies in obstructive sleep apnoea. Eur Respir J. 2011;37(5):1000-28. https://doi.org/10. 1183/09031936.00099710.

23. Malhotra A. Hypoglossal-nerve stimulation for obstructive sleep apnea. N Engl J Med. 2014;370(2): 170-1. https://doi.org/10.1056/NEJMe1314084.

24. Bisogni V, Pengo MF, De Vito A, et al. Electrical stimulation for the treatment of obstructive sleep apnoea: a review of the evidence. Expert Rev Respir Med. 2017;11(9):711-20. https://doi.org/10.1080/ 17476348.2017.1358619.

25. Basyuni S, Barabas M, Quinnell T. An update on mandibular advancement devices for the treatment of obstructive sleep apnoea hypopnoea syndrome. J Thorac Dis. 2018;10(Suppl 1):S48-56. https://doi. org/10.21037/jtd.2017.12.18.

26. Martínez A, Muñiz AL, Soudah E, et al. Physiological and geometrical effects in the upper airways with and without mandibular advance device for sleep apnea treatment. Sci Rep. 2020;10(1):5322. https://doi.org/10.1038/s41598-020-61467-4.

27. Bamagoos AA, Cistulli PA, Sutherland K, et al. Dosedependent effects of mandibular advancement on upper airway collapsibility and muscle function in obstructive sleep apnea. Sleep. 2019a. https://doi. org/10.1093/sleep/zsz049.

28. Lim J, Lasserson TJ, Fleetham J, Wright J. Oral appliances for obstructive sleep apnoea. Cochrane Database Syst Rev. 2004;4:CD004435. https://doi. org/10.1002/14651858.CD004435.pub2.

29. Quinnell TG, Bennett M, Jordan J, et al. A crossover randomised controlled trial of oral mandibular advancement devices for obstructive sleep apnoeahypopnoea (TOMADO). Thorax. 2014;69(10): 938-45. https://doi.org/10.1136/thoraxjnl-2014205464.

30. Gotsopoulos H, Kelly JJ, Cistulli PA. Oral appliance therapy reduces blood pressure in obstructive sleep apnea: a randomized, controlled trial. Sleep. 2004;27(5):934-41. https://doi.org/10.1093/sleep/ 27.5.934.

31. Andrén A, Hedberg P, Walker-Engström ML, Wahlén P, Tegelberg A. Effects of treatment with oral appliance on 24-h blood pressure in patients with obstructive sleep apnea and hypertension: a randomized clinical trial. Sleep Breath. 2013;17(2): 705-12. https://doi.org/10.1007/s11325-012-0746-7.

32. Phillips CL, Grunstein RR, Darendeliler MA, et al. Health outcomes of continuous positive airway pressure versus oral appliance treatment for obstructive sleep apnea: a randomized controlled 
trial. Am J Respir Crit Care Med. 2013;187(8): 879-87. https://doi.org/10.1164/rccm.20121222230C.

33. Bratton DJ, Gaisl T, Wons AM, Kohler M. CPAP vs mandibular advancement devices and blood pressure in patients with obstructive sleep apnea: a systematic review and meta-analysis. JAMA. 2015;314(21):2280-93. https://doi.org/10.1001/ jama.2015.16303.

34. Sharples LD, Clutterbuck-James AL, Glover MJ, et al. Meta-analysis of randomised controlled trials of oral mandibular advancement devices and continuous positive airway pressure for obstructive sleep apnoea-hypopnoea. Sleep Med Rev. 2016;27: 108-24. https://doi.org/10.1016/j.smrv.2015.05. 003.

35. Uniken Venema JAM, Doff MHJ, Joffe-Sokolova D, et al. Long-term obstructive sleep apnea therapy: a 10-year follow-up of mandibular advancement device and continuous positive airway pressure. J Clin Sleep Med. 2020;16(3):353-9. https://doi.org/ $10.5664 /$ jcsm. 8204 .

36. Johal A, Haria P, Manek S, Joury E, Riha R. Readymade versus custom-made mandibular repositioning devices in sleep apnea: a randomized clinical trial. J Clin Sleep Med. 2017;13(2):175-82. https:// doi.org/10.5664/jcsm.6440.

37. Sharples L, Glover M, Clutterbuck-James A, et al. Clinical effectiveness and cost-effectiveness results from the randomised controlled Trial of Oral Mandibular Advancement Devices for Obstructive sleep apnoea-hypopnoea (TOMADO) and longterm economic analysis of oral devices and continuous positive airway pressure. Health Technol Assess. 2014;18(67):1-296. https://doi.org/10.3310/ hta18670.

38. Sutherland K, Dalci O. Fake it till you custom-make it: a non-inferior thermoplastic mandibular advancement device? Thorax. 2019;74(7):629-30.

39. Pépin JL, Raymond N, Lacaze O, et al. Heat-moulded versus custom-made mandibular advancement devices for obstructive sleep apnoea: a randomised non-inferiority trial. Thorax. 2019;74(7):667-74. https://doi.org/10.1136/thoraxjnl-2018-212726.

40. Gindre L, Gagnadoux F, Meslier N, Gustin JM, Racineux JL. Mandibular advancement for obstructive sleep apnea: dose effect on apnea, longterm use and tolerance. Respiration. 2008;76(4): 386-92. https://doi.org/10.1159/000156861.

41. Dieltjens M, Vanderveken OM, Heyning PH, Braem MJ. Current opinions and clinical practice in the titration of oral appliances in the treatment of sleep-disordered breathing. Sleep Med Rev.
2012;16(2):177-85. https://doi.org/10.1016/j.smrv. 2011.06.002.

42. Dort LC, Hadjuk E, Remmers JE. Mandibular advancement and obstructive sleep apnoea: a method for determining effective mandibular protrusion. Eur Respir J. 2006;27(5):1003-9. https:// doi.org/10.1183/09031936.06.00077804.

43. Pételle B, Vincent G, Gagnadoux F, Rakotonanahary D, Meyer B, Fleury B. One-night mandibular advancement titration for obstructive sleep apnea syndrome: a pilot study. Am J Respir Crit Care Med. 2002;165(8):1150-3. https://doi.org/10.1164/ ajrccm.165.8.2108056.

44. Tsai WH, Vazquez JC, Oshima T, et al. Remotely controlled mandibular positioner predicts efficacy of oral appliances in sleep apnea. Am J Respir Crit Care Med. 2004;170(4):366-70. https://doi.org/10. 1164/rccm.200310-1446OC.

45. Remmers J, Charkhandeh S, Grosse J, et al. Remotely controlled mandibular protrusion during sleep predicts therapeutic success with oral appliances in patients with obstructive sleep apnea. Sleep. 2013;36(10):1517-25. https://doi.org/10.5665/ sleep.3048.

46. de Ruiter MHT, Aarab G, de Vries N, Lobbezoo F, de Lange J. A stepwise titration protocol for oral appliance therapy in positional obstructive sleep apnea patients: proof of concept. Sleep Breath. 2020. https://doi.org/10.1007/s11325-020-02045w.

47. Marklund M, Braem MJA, Verbraecken J. Update on oral appliance therapy. Eur Respir Rev. 2019. https://doi.org/10.1183/16000617.0083-2019.

48. Marklund M, Stenlund H, Franklin KA. Mandibular advancement devices in 630 men and women with obstructive sleep apnea and snoring: tolerability and predictors of treatment success. Chest. 2004;125(4):1270-8. https://doi.org/10.1378/chest. 125.4.1270.

49. Sutherland K, Takaya H, Qian J, Petocz P, Ng AT, Cistulli PA. Oral appliance treatment response and polysomnographic phenotypes of obstructive sleep apnea. J Clin Sleep Med. 2015;11(8):861-8. https:// doi.org/10.5664/jcsm.4934.

50. Vecchierini MF, Attali V, Collet JM, et al. Sex differences in mandibular repositioning device therapy effectiveness in patients with obstructive sleep apnea syndrome. Sleep Breath. 2019;23(3):837-48. https://doi.org/10.1007/s11325-018-1766-8.

51. Takaesu Y, Tsuiki S, Kobayashi M, Komada Y, Nakayama H, Inoue Y. Mandibular advancement device as a comparable treatment to nasal continuous 
positive airway pressure for positional obstructive sleep apnea. J Clin Sleep Med. 2016;12(8):1113-9. https://doi.org/10.5664/jcsm.6048.

52. Petri N, Christensen IJ, Svanholt P, Sonnesen L, Wildschiødtz G, Berg S. Mandibular advancement device therapy for obstructive sleep apnea: a prospective study on predictors of treatment success. Sleep Med. 2019;02(54):187-94. https://doi. org/10.1016/j.sleep.2018.09.033.

53. Marques M, Genta PR, Azarbarzin A, et al. Structure and severity of pharyngeal obstruction determine oral appliance efficacy in sleep apnoea. J Physiol. 2019;597(22):5399-410. https://doi.org/10.1113/ JP278164.

54. Vroegop AV, Vanderveken OM, Dieltjens M, et al. Sleep endoscopy with simulation bite for prediction of oral appliance treatment outcome. J Sleep Res. 2013;22(3):348-55. https://doi.org/10.1111/jsr. 12008 .

55. Bamagoos AA, Cistulli PA, Sutherland K, et al. Polysomnographic endotyping to select patients with obstructive sleep apnea for oral appliances. Ann Am Thorac Soc. 2019b;16(11):1422-31. https://doi.org/10.1513/AnnalsATS.201903-190OC.

56. Burgess KR. New insights from the measurement of loop gain in obstructive sleep apnoea. J Physiol. 2012;590(8):1781-2. jphysiol.2012.228643.

57. Sands SA, Edwards BA, Terrill PI, et al. Phenotyping pharyngeal pathophysiology using polysomnography in patients with obstructive sleep apnea. Am J Respir Crit Care Med. 2018;197(9):1187-97. https:// doi.org/10.1164/rccm.201707-1435OC.

58. Terrill PI, Edwards BA, Nemati S, et al. Quantifying the ventilatory control contribution to sleep apnoea using polysomnography. Eur Respir J. 2015;45(2):408-18. https://doi.org/10.1183/ 09031936.00062914.

59. Edwards BA, Andara C, Landry S, et al. Upper-airway collapsibility and loop gain predict the response to oral appliance therapy in patients with obstructive sleep apnea. Am J Respir Crit Care Med. 2016;194(11):1413-22. https://doi.org/10.1164/ rccm.201601-00990C.

60. Petit FX, Pépin JL, Bettega G, Sadek H, Raphaël B, Lévy P. Mandibular advancement devices: rate of contraindications in 100 consecutive obstructive sleep apnea patients. Am J Respir Crit Care Med. 2002;166(3):274-8. https://doi.org/10.1164/rccm. 2008167.

61. Cistulli PA. Oral Appliances In The Treatment of Obstructive Sleep Apnea in Adults. UpToDate https://www.uptodate.com/contents/oralappliances-in-the-treatment-of-obstructive-sleepapnea-in-adults Accessed Oct 10, 2020

62. Clark GT. Mandibular advancement devices and sleep disordered breathing. Sleep Med Rev. 1998;2(3):163-74. https://doi.org/10.1016/s10870792(98)90019-3.

63. Cunali PA, Almeida FR, Santos CD, et al. Mandibular exercises improve mandibular advancement device therapy for obstructive sleep apnea. Sleep Breath. 2011;15(4):717-27. https://doi. org/10.1007/s11325-010-0428-2.

64. Alessandri-Bonetti A, Bortolotti F, Moreno-Hay I, et al. Effects of mandibular advancement device for obstructive sleep apnea on temporomandibular disorders: a systematic review and meta-analysis. Sleep Med Rev. 2019;48:101211. https://doi.org/10. 1016/j.smrv.2019.101211.

65. Teixeira AOB, Andrade ALL, Almeida RCDC, Almeida MAO. Side effects of intraoral devices for OSAS treatment. Braz J Otorhinolaryngol. 2018;84(6):772-80. https://doi.org/10.1016/j.bjorl. 2017.09.003.

66. Patel S, Rinchuse D, Zullo T, Wadhwa R. Long-term dental and skeletal effects of mandibular advancement devices in adults with obstructive sleep apnoea: a systematic review. Int Orthod. 2019;17(1):3-11. https://doi.org/10.1016/j.ortho.2019.01.004.

67. Ngiam J, Balasubramaniam R, Darendeliler MA, Cheng AT, Waters K, Sullivan CE. Clinical guidelines for oral appliance therapy in the treatment of snoring and obstructive sleep apnoea. Aust Dent J. 2013;58(4): 408-19. https://doi.org/10.1111/adj.12111.

68. El-Solh AA, Moitheennazima B, Akinnusi ME, Churder PM, Lafornara AM. Combined oral appliance and positive airway pressure therapy for obstructive sleep apnea: a pilot study. Sleep Breath. 2011;15(2):203-8. https://doi.org/10.1007/s11325010-0437-1.

69. Liu HW, Chen YJ, Lai YC, et al. Combining MAD and CPAP as an effective strategy for treating patients with severe sleep apnea intolerant to highpressure PAP and unresponsive to MAD. PLOS ONE. 2017;12(10):e0187032. https://doi.org/10.1371/ journal.pone.0187032.

70. Vuorjoki-Ranta TR, Kämppi A, Aarab G, et al. (2020) Mandibular advancement device therapy for obstructive sleep apnea: a longitudinal study among patients treated in community dental care in Finland-Potential for the precision medicine approach. Cranio. 2020. https://doi.org/10.1080/ 08869634.2020 .1752431$. 